\title{
51- Auf den Zielwert kommt es an
}

52- Einfache Therapie fördert die Compliance

\section{Pro \& Kontra}

Kontroversen in der Kardiologie

\section{Statintherapie auch bei niedrigem Risiko sinnvoll}

\section{Auf den Zielwert kommt es an}

\author{
GERALD KLOSE
}

Im April dieses Jahres galt eine im JAMA gestartete Diskussion der Indikation von Statinen in primärpräventiver Intention [1]. Dabei ging es um die Frage, ob „ein 55-jähriger, sonst gesunder Mann, systolischer Blutdruck $110 \mathrm{mmHg}$, Gesamtcholesterin $250 \mathrm{mg} / \mathrm{dl}$, familienanamnestisch keine vorzeitige $\mathrm{KHK}^{\text {“ }} \mathrm{mit}$ einem Statin behandelt werden sollte. Argumente, mit denen eine Behandlung bejaht wurde, waren:

- Der zentrale Stellenwert von Cholesterin in der Atherogenese, - ein für den Patienten anzunehmendes 10-Jahres-Risiko für ein koronares Ereignis von 10\% und damit

- eine leitliniengerechte Behandlung mit einem LDL-CholesterinZielwert von $130 \mathrm{mg} / \mathrm{dl}$ oder optional $100 \mathrm{mg} / \mathrm{dl}$ [3].

Als Evidenz für den therapeutischen Nutzen wurden die Studien WOSCOP, AFCAPS/TexCAPS und JUPITER angeführt.

Metaanalyse bestätigt Nutzen auch bei niedrigem Risiko Dass ein relevanter Anteil koronarer Ereignisse auch bei vollkommen leerer Anamnese und gemäß der Algorithmen niedrigem kardiovaskulärem Risiko eintreten, ist ärztlicher Alltag. Dementsprechend hat die neue Metaanalyse der Cholesterol Treatment Trialist's (CTT) große Bedeutung, auch für kommende Leitlinien [6]. Es wird der Behandlungsnutzen auch bei niedrigeren Risiken nachgewiesen, die in den Empfehlungen bislang nicht eingeschlossen waren. Die Auswertung der individuellen Daten von über 130000 Teilnehmern lässt im Gegensatz zu früheren Metaanalysen eine durchaus relevante Risikosenkung für kardiovaskuläre Ereignisse ablesen. Wie bei weiter zurückliegenden Metaanalysen bestätigt sich auch die Sicherheit der Prävention, sodass die Autoren eine Berücksichtigung der niedrigeren Risikoschwelle in kommenden Leitlinien anmahnen.

\section{Zwei mögliche Behandlungsstrategien}

Zwei Behandlungsstrategien spielen in Deutschland eine große Rolle: Es geht darum, entweder Statine in einer festen evidenzbasierten Dosis zu verordnen oder um die Titrierung auf einen vom Risiko abhängigen LDL-Cholesterin-Zielwert, wie er beispielsweise auch in den jüngsten internationalen Leitlinien empfohlen wird [8]. Nach einer interessanten und häufig zitierten
Betrachtung im Lancet von J. Shepherd, dem Principal Investigator der WOSCOP-Studie, stehen sich diese Strategien als „fire and forget" oder "treat to target" gegenüber [9].

\section{Präventives Potenzial nicht verschenken}

Die Tatsache, dass die fixe Dosierung von $40 \mathrm{mg}$ Simvastatin häufig nicht ausreichend ist, um den Zielwert von $<70 \mathrm{mg} / \mathrm{dl}$ oder eine LDL-Cholesterin-Senkung um $>50 \% \mathrm{zu}$ erreichen, macht die Implikation einer solchen Empfehlung klar, nämlich das Verschenken präventiven Potenzials. Die CTT-Metaanalyse $\mathrm{zu}$ intensiverer Behandlung mit Statinen gegenüber Standarddosen belegt zweifelsfrei einen Zusatznutzen mit Erreichung noch niedrigerer, nämlich teilweise $<70 \mathrm{mg} / \mathrm{dl}$ liegender LDLCholesterin-Werte [10].

Die Empfehlung einer höheren, festen Standarddosis eines potenten Statins liegt nicht vor. Gegen sie spricht auch die Möglichkeit der Überbehandlung mit unnötigen Verträglichkeitsproblemen. Die 80-mg-Simvastatin-Dosis wurde in jüngster Zeit auch von regulatorischen Stellen so problematisiert, dass dieser Behandlungsweg praktisch ausgeschlossen ist [11].

Gerne wird die bessere Praktikabilität der Strategie der festen Dosis argumentativ ins Feld geführt. Interessanterweise scheint diese aber gerade eher mit einer schlechteren Compliance verbunden zu sein. Es konnte beim Vergleich mit der Zielwertstrategie gezeigt werden, dass nach einem Jahr bei dieser noch $>60 \%$ Statine einnahmen, während nach Verordnung einer festen Dosis nur noch bei $<10 \%$ Compliance vorlag [12].

\section{Fazit}

Das Pro in der Debatte um die primäre Statin-Behandlungsstrategie hat eindeutig die stärkeren Argumente. Wo liegt das Recht zur Begrenzung der Umsetzung unserer präventiven Möglichkeiten? In der Ökonomie? Dann auch ehrlich so argumentieren!

\section{Korrespondenzadresse:}

Prof. Dr. med. Gerald Klose,

Praxis Dres. Beckenbauer \& Maierhof,

Am Markt 11, 28195 Bremen

E-Mail: klose.bremen@t-online.de 